\title{
The Effect of Mn-Depleted Surface Layer on the Corrosion Resistance of Shape Memory Fe-Mn-Si-Cr Alloys
}

\author{
G. COCCIA LECIS, C. LENARDI, and A. SABATINI
}

\begin{abstract}
In this work, we have examined $\mathrm{Fe}-\mathrm{Mn}-\mathrm{Si}-\mathrm{Cr}$ alloys possessing a good shape memory effect due to a high Mn content ( $28 \mathrm{wt}$ pct). The addition of $\mathrm{Cr}(5 \mathrm{wt}$ pct) was made in order to give fairly good corrosion resistance to the alloy. But we have verified that even in moderately corrosive environments, the presence of chromium does not bear any passivation. On the other hand, we have found that the alloy can acquire corrosion resistance by means of the formation of a Mn-depleted surface layer obtained by heating the alloy at high temperature $\left(\simeq 1050{ }^{\circ} \mathrm{C}\right)$ in air. This modified layer forms since manganese is selectively oxidized with respect to the other components. The adhesion of this layer is maintained even under severe stress if the thickness of the modified region does not exceed $20 \mu \mathrm{m}$. Under this limit, the shape memory characteristics of the alloy are not affected, and at the same time, the specimen acquires properties of passivity comparable with one of the most common austenitic stainless steels at the presence of the same environmental conditions.
\end{abstract}

\section{INTRODUCTION}

THE Fe-based shape memory alloys (SMAs) have undergone a significant development in recent years. ${ }^{[1-6]}$ These low cost materials have large significant applications in different fields, i.e., as thermal alarm actuators, as coupling systems for underground pipes, and in general for many applications requiring materials having a high damping capacity. One of the major problems that limits the use of these alloys is the low corrosion resistance due to the presence of a high percentage of Mn. This element is essential for obtaining a high performance SMA. For the alloy of interest, $\mathrm{Cr}$ has been added in order to stabilize the surface. But we have experimentally verified that there is no improvement with respect to these alloys deficient in chromium. In this work, we present an alternative method for giving corrosion resistance without modifying the basic composition of the alloy. It consists of reducing the Mn content in the surface layer by up to less than 50 pct of its nominal value by means of an air oxidation process at high temperature.

Oh et al..$^{[7,89]}$ have found preferential oxidation of $\mathrm{Mn}$ in Fe-Mn-Si and Fe-Mn-Ni-Si alloys having a Si content of 2 pct. They indicate that the selective oxidation results in the growth of a Mn-depleted layer under the oxide scale. We have verified a similar behavior in an alloy with a higher content of silicon and containing chromium. The concentration of Mn drops gradually across the depleted layer and reaches a minimum at the scale-metal interface. The selective removal of $\mathrm{Mn}$ from the alloy results in the transformation from fcc (austenite) to bcc (ferrite) structure.

The mechanism for preferential oxidation of manganese can be summarized as follows. The $\mathrm{Mn}$ and $\mathrm{Si}$ oxides are more stable than the oxides of the other elements present in the alloy. In the oxide scale forming on the alloy, the $\mathrm{Mn}$ cations are more mobile of the $\mathrm{Si}^{4+}$ and $\mathrm{O}^{2-}$ ions. ${ }^{[7]}$

G. COCCIA LECIS, Professor, C. LENARDI, Researcher, and A. SABATINI, Postdoctoral Student, are with the Department of Applied Physical Chemistry, Politecnico I Milano, I-20133 Milano, Italy.

Manuscript submitted July 16, 1996.
Thus, a layer of Mn oxides growths over a layer of mixed oxides of $\mathrm{Si}, \mathrm{Mn}, \mathrm{Fe}$, and $\mathrm{Cr}$ at the time of oxidation process. The thin silicate layer is almost entirely amorphous. It forms at the interface scale alloy. This layer limits the migration of all the other elements except for $\mathrm{Mn}$, which continues to diffuse toward the outer surface. Thus, the spalling scale is formed by a crystalline part of Mn oxides and by an amorphous part of silicates. At a constant temperature $T$, steady-state conditions are reached at the interface between the crystalline zone and the amorphous zone, between the amorphous zone and the depleted layer, and between the depleted layer and the bulk. As the scale is removed, the Mn-depleted layer reaches conditions of passivity in weakly aggressive environments.

We have also verified that this depleted layer does not substantially affect the shape memory characteristic of this alloy.

\section{EXPERIMENTAL PROCEDURE}

The alloy under investigation was produced by the Nippon Steel Corporation (Chiba, Japan) with the following composition (wt pct): $28.0 \mathrm{Mn}-6.15 \mathrm{Si}-5.16 \mathrm{Cr}$. Alloy specimens were cut from a flat sheet having a thickness of 4 $\mathrm{mm}$ and were subsequently given the following thermal treatment (TT).

(1) Austenizing TT to eliminate the rolled structure and to obtain a homogeneous fcc structure. This treatment consists of a 1-hour hold at $1050{ }^{\circ} \mathrm{C}$, under vacuum $(p$ $=10^{-3} \mathrm{~mm} \mathrm{Hg}$ ), and a furnace cool to provide a minimum cooling velocity. Thus, the fcc structure is stabilized and the onset of the $\varepsilon$ phase (hcp) obtained at room temperature is minimized.

(2) Oxidizing TT is performed by heating the specimen in air at $1050{ }^{\circ} \mathrm{C}$ for periods ranging between 15 and 120 minutes.

The composition and the thickness of the scale and of the depleted layer have been determined by means of scanning electron microscopy (SEM, Cambridge Stereo Scan 360). A semiquantitative X-ray energy dispersive analyzer was used. X-ray counts were calibrated against the bulk com- 


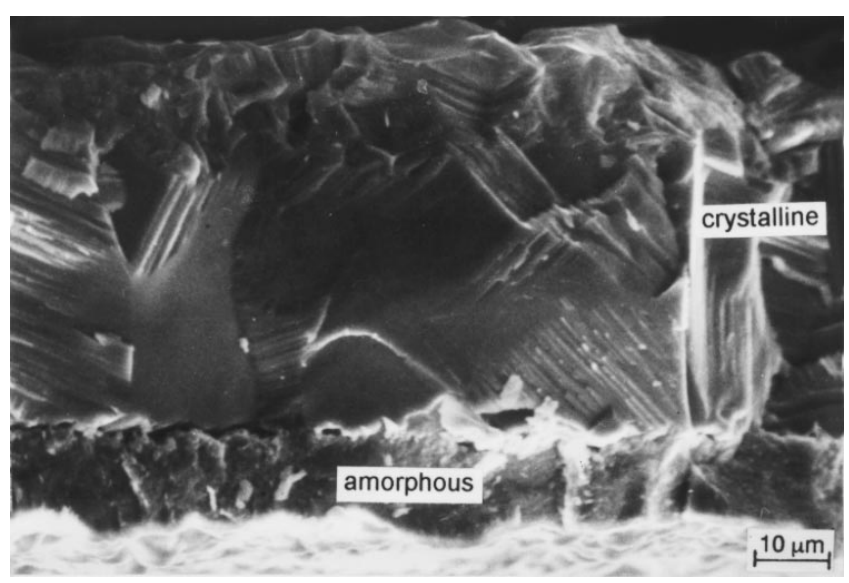

Fig. 1-Scanning electron micrograph of oxide scale. Crystalline layer: 100 pet $\mathrm{Mn}$; and amorphous layer: 40 pct $\mathrm{Mn}, 5 \mathrm{pct} \mathrm{Si}$, and 55 pet $\mathrm{Cr}$.

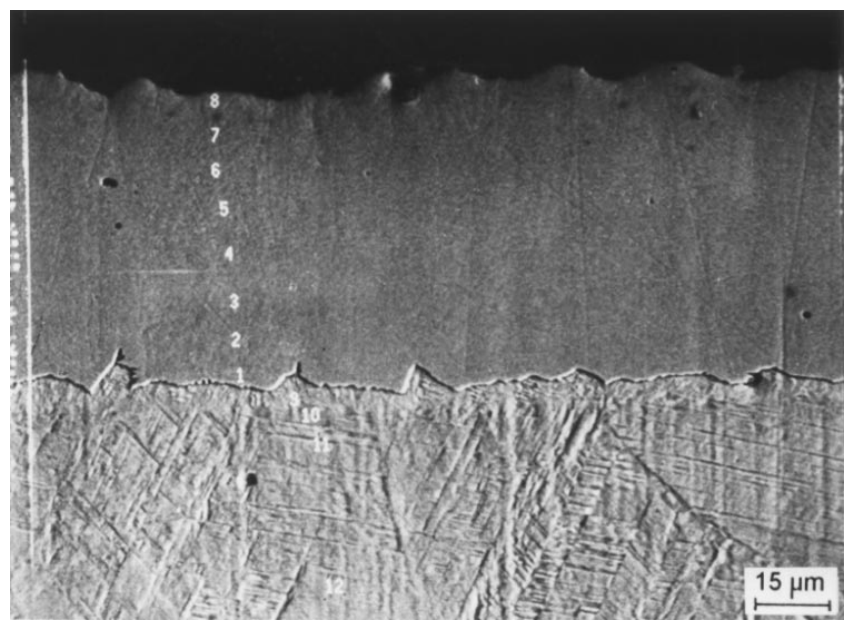

Fig. 2-Scanning electron micrograph of the metal after spalling of the scale. On the top: the Mn depleted layer; and on the bottom: the bulk. The numbers indicate the positions where the elemental concentrations have been evaluated. The martensitic structure of the bulk has been induced by the mechanical polishing of the transverse section of the specimen.

position of the nominal alloy as given by the Nippon Steel Corporation. The crystalline structure has been resolved by using an X-ray diffractometer (PHILIPS* PW 1830). A

*PHILIPS is a trademark of Philips Electronic Instruments Corp., Mahwah, NJ.

Vicker's indenter (Microindenter, Shimadzu HV 2000) was used for microhardness measurements. The electrochemical behavior has been evaluated by potentiodynamic measurements. They have been performed by using an EG\&G potentiostat/galvanostat and an ASTM cell with calomel reference electrode and two Pt counterelectrodes. Experimental curves have been obtained with an induced voltage ranging between -200 and $1800 \mathrm{mV}$ with respect to the open circuit potential. Tests have been carried out at room temperature in the presence of sulfate ions. The base solution was $\mathrm{Na}_{2} \mathrm{SO}_{4}(0.05 \mathrm{M})$, and the $\mathrm{pH}$ was varied by adding $\mathrm{H}_{2} \mathrm{SO}_{4}$ or $\mathrm{NaOH}$. The effect of $\mathrm{Cl}^{-}$ions (1000 ppm) due to $\mathrm{NaCl}$ additions has been analyzed. In order to evaluate the shape memory performance, several bending tests were performed. Recovery measurements were made on a

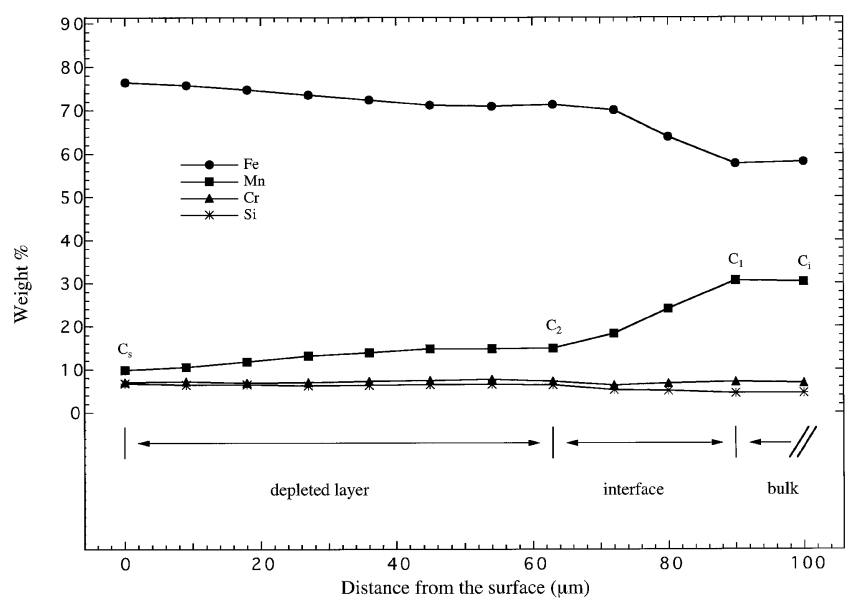

Fig. 3-Semiquantitative microanalysis of the alloy from the Mn-depleted layer into the bulk. Three zones are evident: the depleted layer, the interface between the depleted layer and the bulk, and the bulk.

sample by repeating the following procedure: (1) bending to $30 \mathrm{deg}$ at room temperature and (2) annealing at $400{ }^{\circ} \mathrm{C}$ for 15 minutes.

\section{RESULTS AND DISCUSSION}

The typical effect of high-temperature oxidation of the specimen after 1-hour thermal treatment is the formation of an oxide scale which spontaneously spalls from the specimen. In Figure 1, the SEM image shows the scale constituted of an amorphous layer at the scale-metal interface and of an external crystalline layer. The amorphous layer has a thickness of 8 to $9 \mu \mathrm{m}$, whereas the crystalline layer has one of about $50 \mu \mathrm{m}$. X-ray diffraction measurements reveal that the first layer is composed of nanocrystals of Mn oxides (principally $\mathrm{MnO}$ ) and of $\mathrm{Mn}-\mathrm{Fe}-\mathrm{Cr}$ mixed oxides embedded in an amorphous matrix of silicates, and the crystalline layer is only composed of Mn oxides (almost entirely of $\mathrm{Mn}_{2} \mathrm{O}_{3}$ ). This structure indicates that the oxidation takes place by means of a selective migration process of Mn toward the outer surface. After spalling of the scale, a surviving thin layer $(\simeq 1 \mu \mathrm{m})$, principally composed of $\mathrm{Fe}$ and $\mathrm{Mn}$ oxides, sticks to the metal and can be removed by mechanical polishing.

Figure 2 shows an SEM micrograph illustrating the morphological change within 70 to $80 \mu \mathrm{m}$ of the surface layer without the scale after polishing. A detailed elemental mapping was made on a cross section from the surface to that of the bulk. The analysis gives the composition and the variation of the structure down to the depth at which the relative concentrations approached the original alloy. In Figure 3 , the semiquantitative microanalysis results are shown: the concentration of Mn ranges between $9 \mathrm{wt}$ pct at the external surface and 28 wt pct in the bulk. X-ray diffraction measurements reveal the bcc structure of the depleted layer having a value of the lattice parameters very similar to that of the $\alpha$-ferrite in Fe-Mn system. This layer does not present any martensitic transformation. The internal grain structure is very regular; this is shown in Figure 4. The grain size as expected is concomitant with the extended time at temperature conditions. In Figure 5, the hardness values across the depleted layer are reported. The 


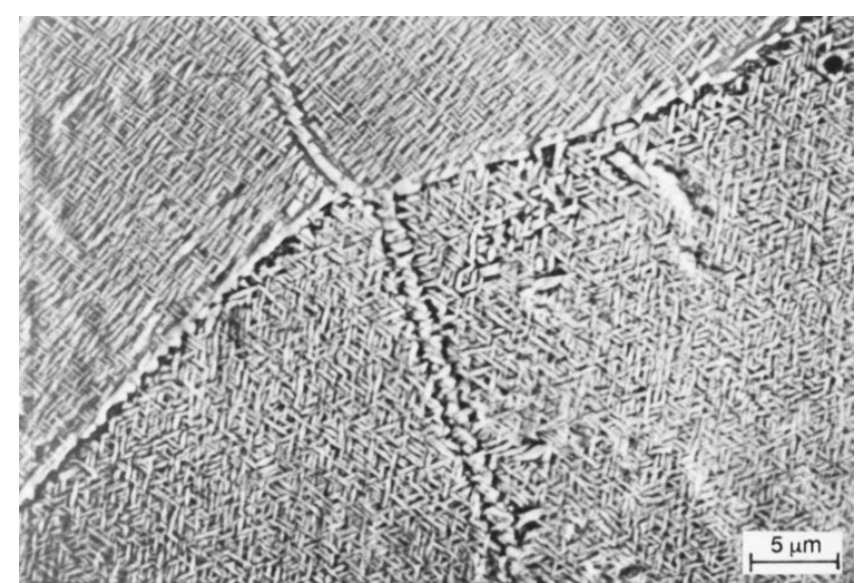

Fig. 4-The internal grain structure of the shape memory Fe-Mn-Si-Cr alloy.

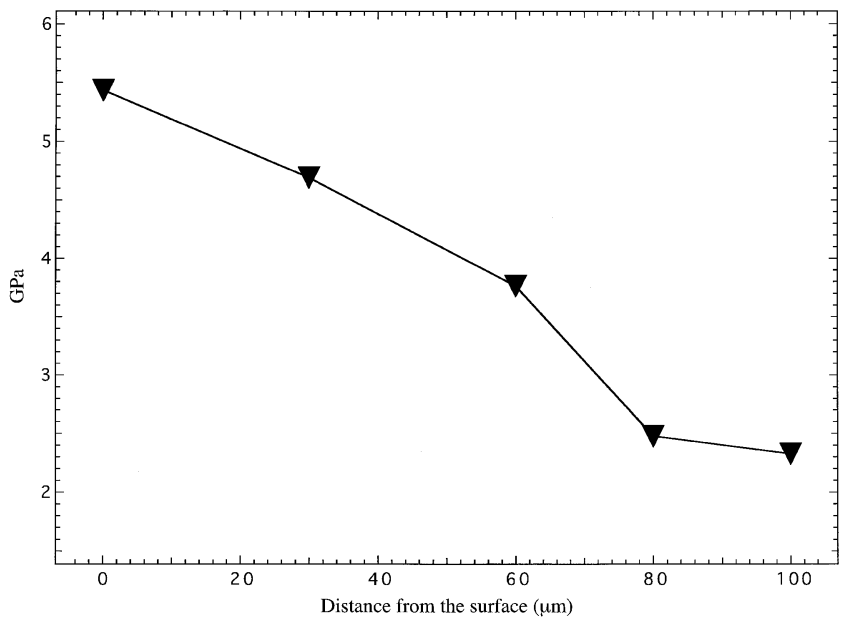

Fig. 5-Hardness of the alloy from the surface of the depleted layer into the bulk.

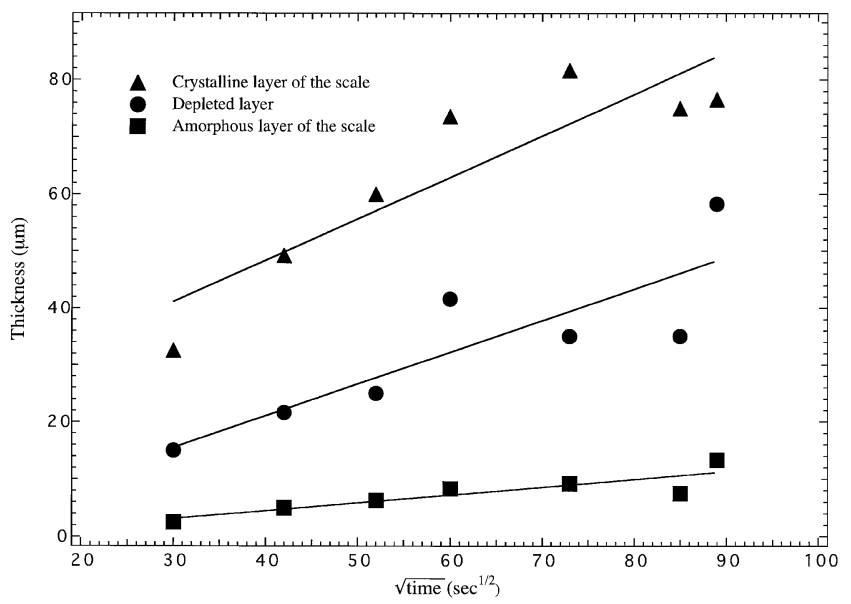

Fig. 6- Growth rate of the crystalline and of the amorphous layers of the scale and of the Mn-depleted layer. The straight lines are least-squares fit to each experimental curve.

layer is harder (5.5 GPa) than the bulk (2.2 GPa). To obtain more information on the kinetics of the phenomena correlated with the oxidation process, a series of thermal treatments of varying durations were performed. It was observed that the Mn percentages near the scale and near the bulk are sufficiently stable. The only factor that changes with the exposure time to an oxidizing atmosphere is the thickness of the depleted layer. Oh et al. ${ }^{[7,8,9]}$ have obtained similar results in oxidation processes of $\mathrm{Fe}-\mathrm{Mn}-\mathrm{Si}$ alloys having an fcc structure and a Si content of $<2$ pct. In agreement with these authors, we have observed that in the early stage of oxidation, the oxides of all three metals form and the specimen evolves in the following way.

(1) Due to the high Si content, an amorphous layer of silicates forms from the beginning (after only 15 minutes under oxidation conditions) and it constitutes the inner part of the scale. This gives rise to a barrier permeable to only Mn, which, when it reaches the external surface, oxidizes and forms the crystalline portion of the scale.

(2) The Mn oxidation takes place on the external surface of the scale, and consequently, a Mn-depleted layer forms on the alloy. Thus, the depleted layer changes its structure, i.e., from fcc to bcc, as it can be inferred from the Fe-Mn phase diagram.

The experimental data indicate that the growth of the three layers, i.e., crystalline and amorphous part of the scale and the depleted layer, follows a law of the type

$$
H=k \sqrt{t}
$$

where $H$ is the thickness of the layer, $k$ is a constant, and $t$ is the time of oxidation. Figure 6 shows the data in good agreement with the Eq. [1] within a few minutes $(\simeq 15$ minutes) after the beginning of the oxidation process. The crystalline part of the scale and the depleted layer have very similar growth rates. This is consistent with the fact that only Mn diffuses through the bcc (depleted) layer. The growth of the amorphous part of the scale has a much lower rate, perhaps due to the fact that the concentrations of $\mathrm{Cr}$ and $\mathrm{Mn}$ tend to reach their saturation values. In order to determine the diffusion coefficient of Mn through the depleted layer, it has been assumed that the driving force in Mn migration is the concentration gradient that tends to form within the layer. If the concentrations of $\mathrm{Mn}$ at the outer surface of the scale and at both sides of the depleted layer are constant, as in our case, Eq. [1] can be written as

$$
H=2 \beta \sqrt{D_{\text {dep } 1} \Delta t}
$$

where $D_{\text {depl }}$ is the diffusion coefficient of $\mathrm{Mn}$ in the bcc layer, $\Delta t$ the duration time at $1050{ }^{\circ} \mathrm{C}$, and $\beta$ a dimensionless constant which satisfies the following relation:

$$
C_{2}-C_{1}=\frac{C_{s}-C_{2}}{\sqrt{\pi} \beta e^{\beta^{2}} \operatorname{erf}(\beta)}-\frac{C_{1}-C_{i}}{\sqrt{\pi} \beta e^{\beta^{2}} \operatorname{erfc}(\beta \sqrt{\phi})}
$$

where $C_{1}$ is the concentration of $\mathrm{Mn}$ at the interior side of the interface between the depleted layer and the bulk, $C_{2}$ the concentration of $\mathrm{Mn}$ at the exterior side of the interface between the depleted layer and the bulk, $C_{i}$ the concentration of $\mathrm{Mn}$ in the bulk, and $C_{s}$ the concentration of $\mathrm{Mn}$ at the scale-depleted layer interface (Figure 2). The coefficient of diffusion of $\mathrm{Mn}$ in the bulk layer is given by $D_{\text {bulk }}$ and $\phi$ is given by $D_{\text {depl }} / D_{\text {bulk }}$. Thus, Eq. [3] can be solved by imposing appropriate simplifications. In particular, it has been assumed that $C_{1}=C_{i}$, that is, we have disregarded the migration of $\mathrm{Mn}$ in the bulk. The values of the diffusion coefficient evaluated for the different thermal treatments are about $10^{-8} \mathrm{~cm}^{2} / \mathrm{s}$. Using these values, the results are two orders of magnitude lower than those determined for an 


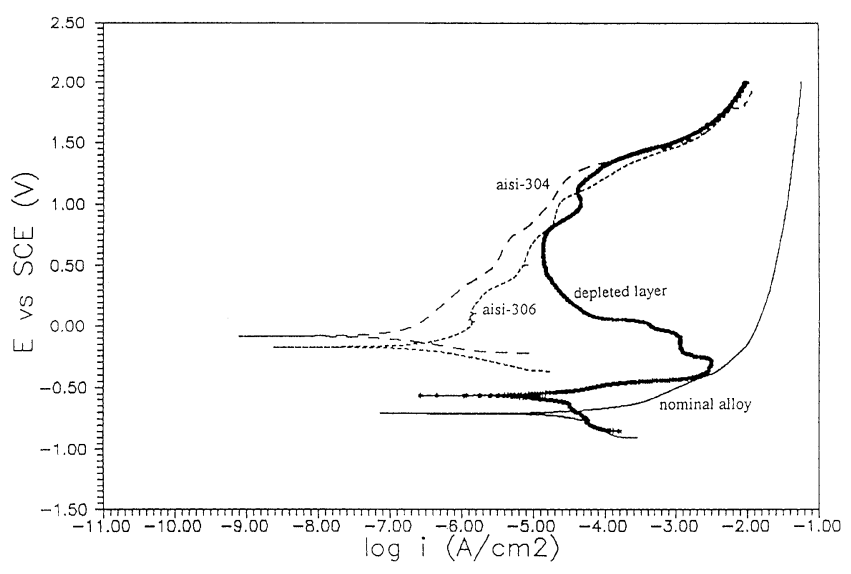

Fig. 7-Potentiodynamic curves at $\mathrm{pH} 6$ of the nominal alloy, of the alloy with the depleted layer, and of two types of stainless steels (AISI-304 and AISI-306).

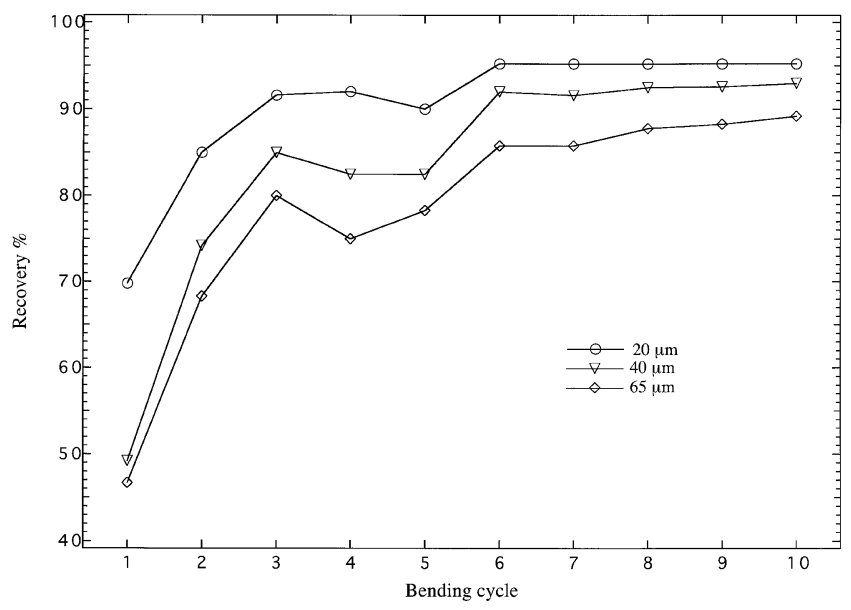

Fig. 8-Recovery comparisons as a function of the bending cycle for different thicknesses of the depleted layer.

alloy having an equivalent percentage of $\mathrm{Mn}$, however, without any Cr. A smaller diffusion coefficient can be argued as a consequence of a lower slope of the Mn concentration curve in the interface region (Figure 3) with respect to the curve reported by $\mathrm{Oh}$ et al. ${ }^{[7]}$ This difference could be due to the similar atomic size of the two elements: $\mathrm{Cr}$ would tend to occupy vacancies in the lattice reducing the available sites for Mn migration.

In order to verify that the decreased concentration of $\mathrm{Mn}$ in the depleted layer gives a higher stability of the surface with respect to the nominal alloy, potentiodynamic measurements and immersion tests were performed. Figure 7 shows the anodic polarization curves at $\mathrm{pH} 6$ of the original alloy as well as that for the alloy with the depleted layer. For comparison, the curves of two types of stainless steels (AISI-304 and AISI-306) under similar experimental conditions are also shown. It is observed that the presence of a depleted layer produces a more noble alloy as it is shown by the increase of the open circuit potential up to about $\simeq 100 \mathrm{mV}$ when compared to the nominal alloy composition. At higher applied potential, the data are similar to one of the traditional stainless steels (AISI-306). In neutral or weakly alkaline environments ( $\mathrm{pH} 6$ to 10), the corrosion penetration rate is about $100 \mu \mathrm{m}$ per year. In these condi- tions, the Mn-depleted layer favors the processes of passivation. On the other hand, under acidic conditions, as in the case of the presence of $\mathrm{Cl}$ ions, the behavior of the alloy with the depleted layer is as active as that of the bulk.

The bend tests prove simultaneously that a good shape recovery and adhesion to the bulk are maintained if the depleted layer thickness is about $20 \mu \mathrm{m}$. This corresponds to a minimal value for maintaining the corrosion resistance previously described. Figure 8 shows a comparison between specimen recoveries having different thicknesses of the modified layer after bending to $30 \mathrm{deg}$ with a subsequent anneal at $400{ }^{\circ} \mathrm{C}$ for 15 minutes. A more complete and immediate shape recovery is achieved by applying a predeformation of $30 \mathrm{deg}$ and subsequent bending cycles of $15 \mathrm{deg}$.

\section{CONCLUSIONS}

In this work, the kinetics of oxidation for a Fe-Mn-Si$\mathrm{Cr}$ alloy has been studied. The formation of a Mn-depleted layer is favored by the high silicon content which, during the high-temperature oxidizing process, creates a barrier permeable for only $\mathrm{Mn}$ at the scale-metal interface. The concentration of all the elements at both sides of the depleted layer is constant for each layer thickness, which depends on the duration of the oxidizing process. The passivation behavior of the depleted layer has been verified in different aqueous environments. The constant concentrations of the elements guarantee the property of passivation for this kind of alloy undergoing thermal treatment for oxidation.

The shape recovery of the oxidized specimen is comparable to that of the nominal alloy, provided that the thickness of the depleted layer does not exceed $20 \mu \mathrm{m}$.

This kind of SMA modified by the oxidation process is a good candidate for SMA systems requiring corrosion resistance in moderately aggressive environments.

\section{ACKNOWLEDGMENT}

The work has been carried out under the financial support of the Ministero dell'Università e della Ricerca Scientifica e Tecnologica (MURST).

\section{REFERENCES}

1. A. Sato, E. Chisima, K. Soma, and T. Mori: Acta Metall., 1982, vol. 30 , pp. 1177-83.

2. M. Murakami, H. Otsuka, H.G. Suzuki, and S. Matsuda: Proc. of Int. Conf. on Martensitic Transformation, The Japan Institute of Metals, Nara, Japan, 1986, pp. 985-90.

3. I.N. Bogachev, G.Ye. Zvigintseva, V.F. Yegolayev, and G.I. Lyapunov: Fiz. Metall. Metalloved., 1969, vol. 28, p. 1018.

4. H. Umebayashi and Y. Ishikawa: J. Phys. Soc. Jpn., 1966, vol. 21, p. 1281.

5. O.A. Khomenko, I.F. Khilkevich, and G.Ye. Zvigintseva: Fiz. Metall. Metalloved., 1974, vol. 37, p. 1325.

6. Yu.N. Makogen and B.I. Nikolin: Fiz. Metall. Metalloved., 1973, vol. 37, p. 864.

7. J.M. Oh, J. McNallan, and W.E. King: J. Electrochem. Soc., 1986, vol. 133 (5), pp. 1042-48.

8. J.M. Oh and J. McNallan: Corrosion-Nace, 1987, vol. 43, pp. 561-63.

9. J.M. Oh and J. McNallan: J. Electrochem. Soc., 1987, vol. 134 (4), pp. 1010-15. 